\title{
Hybrid ameloblastoma of the maxilla: A case report and review of literature
}

\section{Case Report}

\author{
Lydia Melek and Mona S. Oraby \\ Department of Oral and Maxillofacial, Faculty of Dentistry, Alexandria University, \\ Alexandria, Egypt
}

\begin{abstract}
The desmoplastic variant is a rare form which represents about $4-5 \%$ of all ameloblastomas. A few number of studies in the literature have reported "hybrid lesions" which show features of both desmoplastic and conventional ameloblastoma. In this report, we introduce a novel case of hybrid desmoplastic/follicular ameloblastoma of the maxilla, presenting its clinical, radiographic and histopathological features. It has been reported that the desmoplastic variant has a more aggressive behavior than conventional ameloblastoma, owing to its predilection to expand into a large size when affecting the maxilla and the tendency to early invade the surrounding structures

Despite the rarity of desmoplastic ameloblastoma and "hybrid ameloblastoma", they should be included in the differential diagnosis of slowly growing lesions especially those in the anterior and premolar regions of the jaw. Careful radiographic investigation and accurate histopathological examination of properly taken incisional biopsies will help in providing patients with the best therapeutic option and avoiding waste of time and cost associated with misdiagnosis.
\end{abstract}

Key Words: Ameloblastoma, Desmoplastic, Hybrid ameloblastoma, Follicular ameloblastoma, Psychology.

Received: 31 March 2020, Accepted: 12 May 2020.

Corresponding Author: Lydia Melek, Department of Oral and Maxillofacial, Faculty of Dentistry, Alexandria University, Alexandria, Egypt, Tel.: +2034868308, Mobile: +201224807468, E-mail: lydia.nabil@dent.alex.edu.eg.

ISSN: 2090-097X, October 2019, Vol. 10, No. 4

\section{INTRODUCTION}

Ameloblastoma is an epithelial benign odontogenic tumor with locally aggressive behavior, the desmoplastic variant being considered as a rare form which represents about $4-5 \%$ of all ameloblastomas ${ }^{[1]}$. This histological variant has first been described by Eversole in $1984^{[2]}$.

Desmoplastic ameloblastoma is characterized radiographically by ill-defined borders in contrast to other ameloblastoma variants, reflecting the more aggressive nature of such lesion. This feature accounts for the possibility of its being mistaken for a benign fibro-osseous lesion $^{[1 \text { and } 3]}$.

A few number of studies in the literature have reported "hybrid lesions" which show features of both desmoplastic and conventional ameloblastoma. Hybrid lesions are rare, accounting for about 1.1 to $4.3 \%$ of all ameloblastomas $^{[4-8]}$. In this report, we introduce a novel case of hybrid desmoplastic/follicular ameloblastoma of the maxilla, presenting its clinical, radiographic and histopathological features.

\section{CASE REPORT}

A male patient, 36 years old came to the outpatient clinic of the Oral and Maxillofacial Surgery Department, Faculty of Dentistry, Alexandria University, Egypt, complaining from a maxillary, slowly growing swelling causing pain and numbness at the right cheek that started 7 years ago. During this period, the patient was mistakenly diagnosed by dentists (due to the presence of multiple carious teeth associated with bad oral hygiene) as having a periapical infection and inflammation and was treated accordingly.

Clinical examination has revealed a non-tender swelling extending from the region of the maxillary right lateral incisor to the maxillary right first molar tooth, measuring about $6 \mathrm{~cm}$ in the mesio-distal direction and about $4 \mathrm{~cm}$ in the supero-inferior direction (Figure 1). The swelling was covered by non-ulcerated mucosa, and no lymph nodes were palpable.

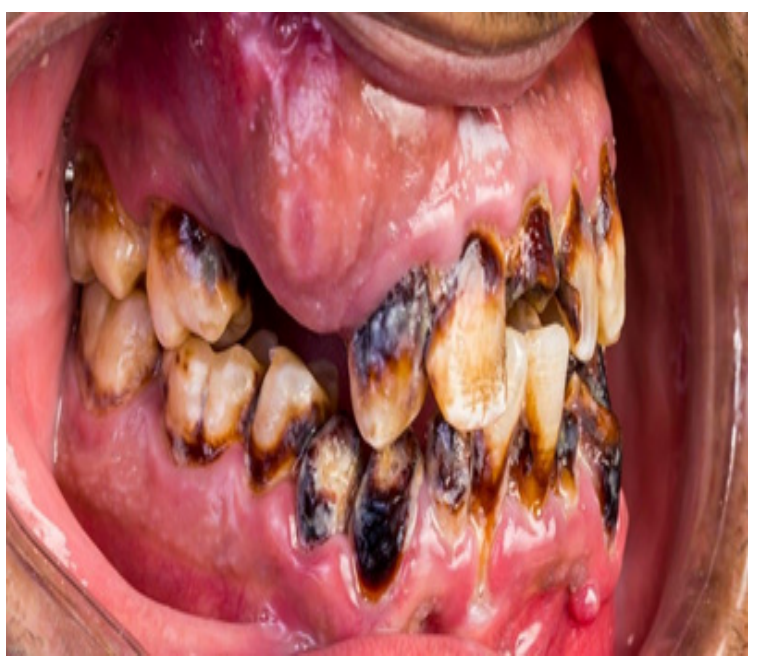

Figure 1: Clinical presentation of the lesion. 
The orthopantomogram has revealed a multilocular radiolucent lesion with somewhat ill-defined borders extending from the maxillary right lateral incisor region to the maxillary right first molar region (Figure 2). On CT examination, the lesion was seen to extend more extensively across the midline from the maxillary left lateral incisor to the mesial surface of the maxillary right first molar, and extending upwards to the nasal floor, together with buccal resorption and palatal expansion (Figures 3 and 4).

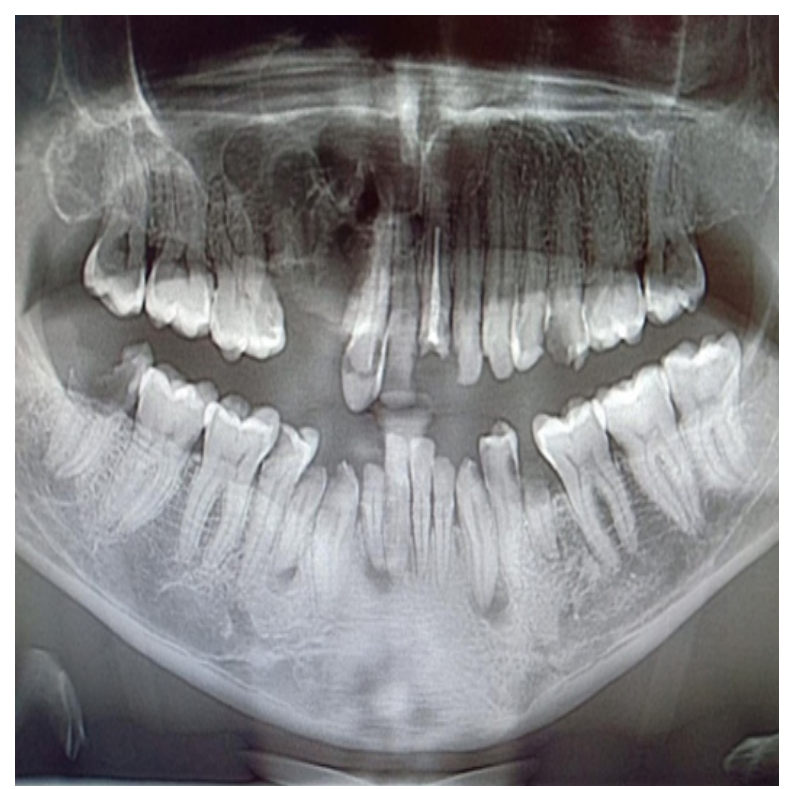

Figure 2: OPG showing soap bubble multilocular radiolucency in the right maxilla and crossing the midline.
An incisional biopsy was performed and the histopathological examination revealed a mixed follicular/ desmoplastic variant of ameloblastoma with cystic change and focal squamous metaplasia.

The treatment plan was to surgically excise the whole lesion with safety margin, from the maxillary left canine to the distal surface of the maxillary right first molar region with scarification of the buccal mucosa and involved teeth. A maxillary vestibular incision was done and the Stryker saw was used to excise the lesion after marking the boundaries of resection with a surgical bur. Preservation of the palatal mucosa was decided in addition to scrubbing of the inner surface of the preserved mucosa using a large round bur in the area opposite to the bony erosion. Separation of the lesion and resected segment from the nasal floor was done safely without any perforation to the nasal mucosa (Figure 5). Suturing of the wound was done on both sides of the defect resulting from the resected lesion. An immediate surgical obturator had been prepared according to the plan and was applied intraoperatively with the aid of soft liner to obturate the surgical defect and it was secured with clasps on the remaining teeth.

Upon follow up, the patient reported that numbness has disappeared, however, complained from mild tingling along the distribution of the infraorbital nerve which decreased gradually until recovered after one month. Also, the bony defect resulting from the resected lesion decreased in size and granulation tissue started to fill the cavity. The surgical obturator was replaced with an intermediate obturator 4 weeks after the operation.
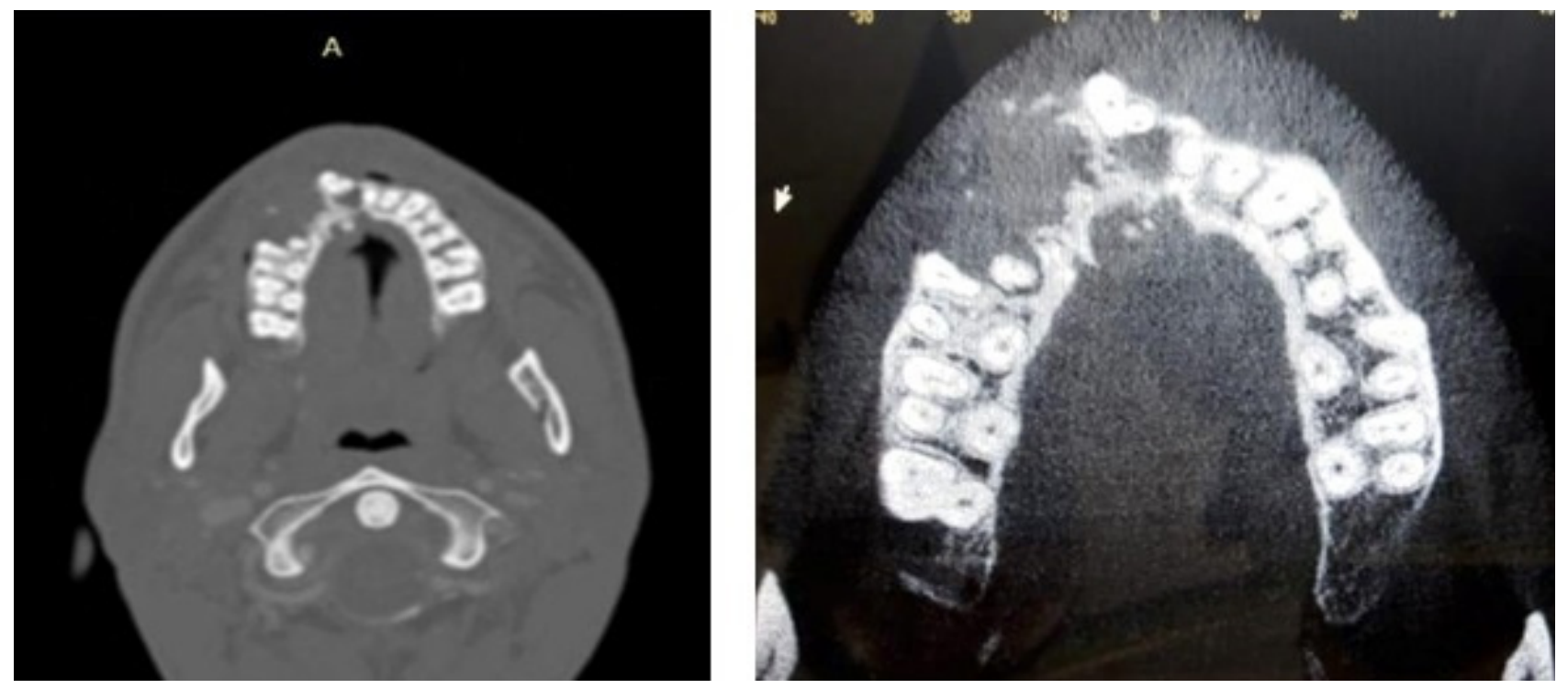

Figure 3: Axial CT section showing the lesion with buccal resorption. 


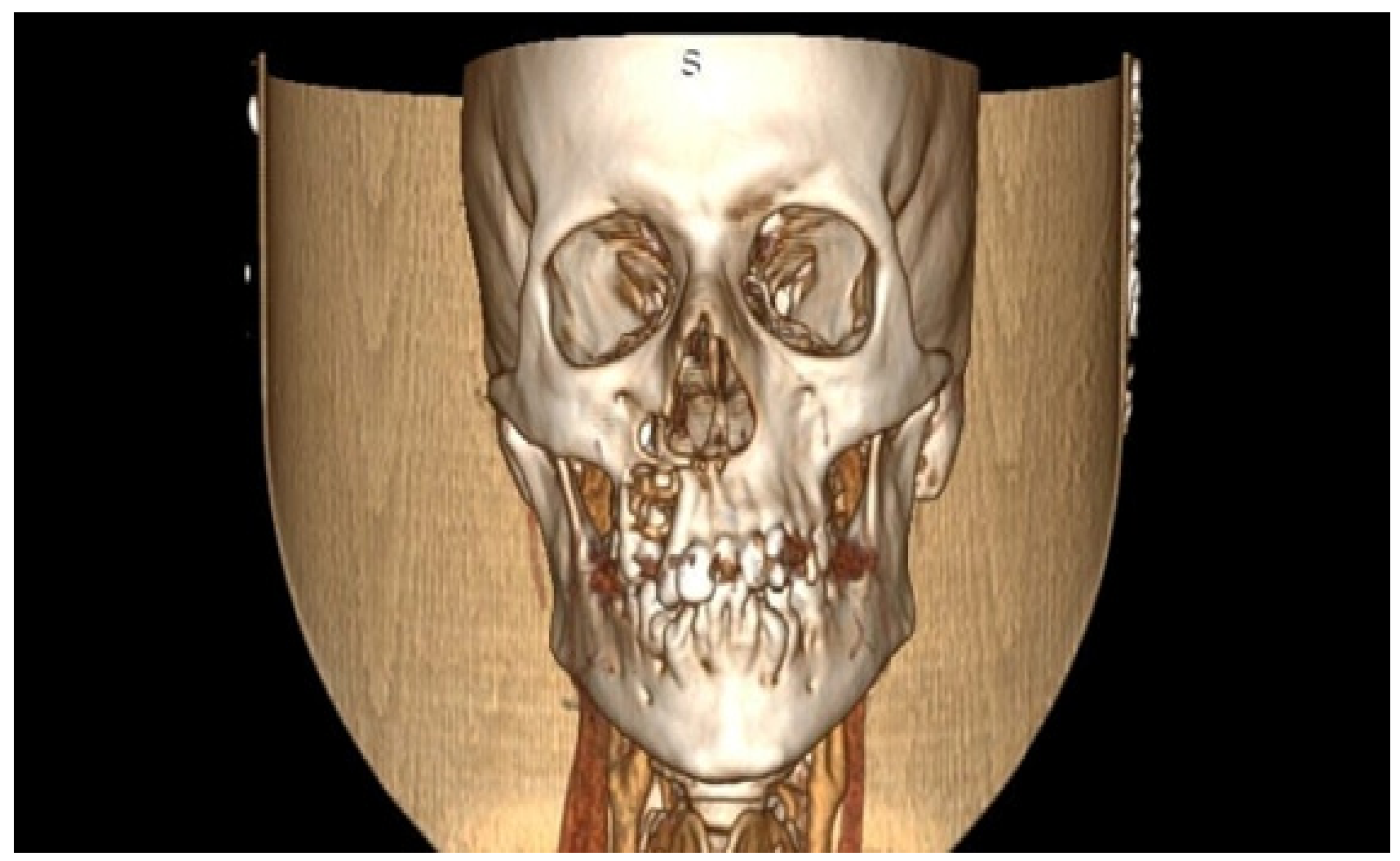

Figure 4: 3D CT showing resorption of the maxillary bone on the right side with upward extension towards the nasal floor.

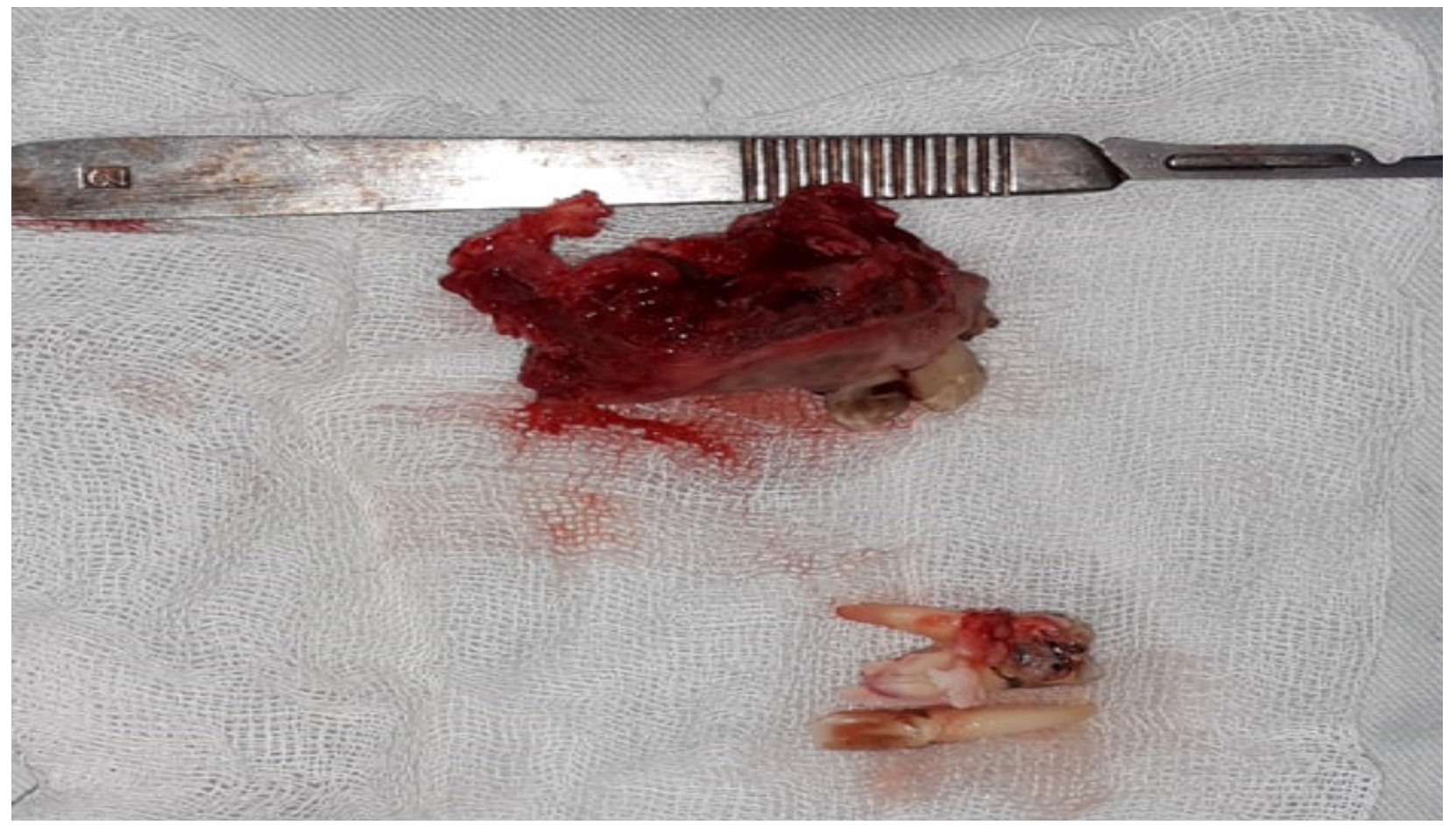

Figure 5: The resected lesion and involved teeth.

The patient has now been clinically followed up for 6 months and uneventful healing was evident. An informed consent has been signed by the patient for publication of the current case report.

\section{DISCUSSION}

Hybrid lesions of ameloblastoma were first described by Waldron and El-Mofty in $1987^{[9]}$. 
Thirty years later, in 2017, Iwase et $a l^{[6]}$ have reviewed the 35 cases of hybrid ameloblastoma reported in the English literature up to the time of their article publication. They found that the mean age of occurrence was 44.9 years with female predominance $(61 \%)$. Our case was a male patient, 36 years of age, slightly younger than the mean age reported in the review. $83 \%$ of cases in their review were found in the mandible, unlike our case which affected the maxilla. $40 \%$ of the reviewed lesions appeared radiographically as mixed radioluceny and radiopacity while $50 \%$ appeared as radiolucencies. $93 \%$ of the reviewed cases were a mixture of desmoplastic and follicular ameloblastoma consistent with the histopathological picture of our presented case.

Desmoplastic ameloblastoma is characterized by a unique histopathological feature that distinguishes it from conventional ameloblastoma, which is the presence of extensive dense collagenous stroma compressing the islands of odontogenic epithelium ${ }^{[6]}$. This was evident in the present case in which parts of the tumor expressed dense sclerotic connective tissue stroma with abundance of collagen fibers, confirming the diagnosis of mixed desmoplastic/follicular ameloblastoma. The nests and islands of odontogenic epithelium were made up of peripherally located palisading ameloblastic cells and centrally located stellate cells. Many nests were undergoing cystic change while others showed focal squamous metaplastic change.

It is not clear in hybrid lesions if desmoplastic changes occur in conventional ameloblastoma or conversely, parts of desmoplastic ameloblastoma change into conventional type ${ }^{[6]}$.

It has been reported that the desmoplastic variant has a more aggressive behavior than conventional ameloblastoma, owing to its predilection to expand into a large size when affecting the maxilla and the tendency to early invade the surrounding structure ${ }^{[10]}$. That is why we considered resection as the optimal surgical option for our case to reduce the chances of recurrence.

\section{CONCLUSION}

Despite the rarity of desmoplastic ameloblastoma and "hybrid ameloblastoma", they should be included in the differential diagnosis of slowly growing lesions especially those in the anterior and premolar regions of the jaw. Careful radiographic investigation and accurate histopathological examination of properly taken incisional biopsies will help in providing patients with the best therapeutic option and avoiding waste of time and cost associated with misdiagnosis.

\section{Funding}

This research did not receive any specific grant from funding agencies in the public, commercial, or not-forprofit sectors.

\section{CONFLICT OF INTEREST}

The authors declare no conflict of interest.

\section{REFERENCES}

1. Kaffe I, Buchner A, Taicher S. Radiologic features of desmoplastic variant of ameloblastoma. Oral Surg Oral Med Oral Pathol 1993; 76: 525 - 529.

2. Eversole LR, Leider AS, Hansen LS. Ameloblastomas with pronounced desmoplasia. J Oral Maxillofac Surg 1984; 42: 735 - 740.

3. Li B, Long $\mathrm{X}$, Wang $\mathrm{S}$, Cheng $\mathrm{Y}$, Chen $\mathrm{X}$. Clinical and radiologic features of desmoplastic ameloblastoma. J Oral Maxillofac Surg 2011; 69: $2173-2185$.

4. Philipsen HP, Reichart PA, Takata T. Desmoplastic ameloblastoma (including "hybrid" lesion of ameloblastoma). Biological profile based on 100 cases from the literature and own files. Oral Oncol 2001; 37: 455 - 460 .

5. Chaitanya B, Chhaparwal Y, Pai KM, Kudva A, Cariappa KM, Acharya S. Hybrid ameloblastoma: An amalgam of rare and conventional ameloblastoma. Contemp Clin Dent 2016; 7: 90 - 94.

6. Iwase $\mathrm{M}$, Fukuoka A, Tanaka $\mathrm{Y}$, Saida N, Onaka E, Bando S, Kondo G. Hybrid Desmoplastic/Follicular Ameloblastoma of the Mandible: A Case Report and Review of the Literature. Case Rep Pathol 2017; 2017: 7031414.

7. Lakshmi CR, Bhavana SM, Nallamilli SM, Prabhat MP, Sarat G, Anuradha C. Hybrid Ameloblastoma of the Maxilla: A Puzzling Pathology. Iran J Med Sci 2016; 41: 340 - 344.

8. Takata T, Miyauchi M, Ogawa I, Zhao M, Kudo Y, Sato S, Takekoshi T, Nikai H, Tanimoto K. So-called 'hybrid' lesion of desmoplastic and conventional ameloblastoma: report of a case and review of the literature. Pathol Int 1999; 49: 1014 - 1018.

9. Waldron CA, el-Mofty SK. A histopathologic study of 116 ameloblastomas with special reference to the desmoplastic variant. Oral Surg Oral Med Oral Pathol 1987; 63: 441 - 451.

10. Mintz S, Velez I. Desmoplastic variant of ameloblastoma: report of two cases and review of the literature. $\mathrm{J}$ Am Dent Assoc 2002; 133: 1072 - 1075. 